Themenheft Nr. 40: CoViD-19 und die digitale Hochschulbildung. Irritationen, Einsichten und Programmatiken

Herausgegeben von Markus Deimann, Marios Karapanos und Klaus Rummler

\title{
Erleben, Herausforderungen und zukünftige Lehrszenarien in der Online-Lehre
}

\section{Eine Mixed-Method-Studie zum Covid-19 Sommersemester 2020 aus Sicht von Lehrenden}

\author{
Sarah Malewski ${ }^{1}$ D, Sabrina Engelmann ${ }^{1}$ (D) und Lars Peppel ${ }^{1}$ \\ ${ }^{1}$ Frankfurt University of Applied Sciences
}

\begin{abstract}
Zusammenfassung
Das SoSe 2020 hat Lehrende vor die Herausforderung gestellt, ihre Lehrveranstaltungen innerhalb kürzester Zeit komplett digital gestützt durchzuführen. Wie Lehrende mit dieser Veränderung umgegangen sind, welche Lehrszenarien sie praktiziert haben, welche Potentiale und Herausforderungen sie in reiner Online-Lehre sehen und wie sie sich Lehre in Zukunft vorstellen, wurde an der Frankfurt University of Applied Sciences mittels eines Mixed-Method-Ansatzes mit Leitfadeninterviews und einem Online-Fragebogen analysiert. In der Gesamtschau lässt sich sagen, dass die Lehrenden trotz der plötzlich auftretenden Corona-Situation die Lehre im SoSe 2020 insgesamt positiv erlebt haben. So berichten sie von einem grossen Kompetenzzuwachs, was etwa die Interaktion und Kommunikation über Videokonferenzsysteme oder das Erstellen von Medienprodukten angeht. Die Grundpfeiler des Lehrkonzepts bildeten bei vielen Lehrenden zu Beginn der Pandemie eine Kombination von Videokonferenzen, vorab aufgezeichneten Lehrvorträgen und Lehrmaterialien, die über Lernplattformen zur Verfügung gestellt wurden. Zentrale Herausforderungen waren dabei die fehlende Beziehungsebene und die sinnvolle Gestaltung von Gruppenarbeiten. Die grösstenteils positiven Erfahrungen des SoSe 2020 haben die Bereitschaft der Lehrenden erhöht, in Zukunft eine Kombination aus Präsenz- und Online-Lehre anzubieten bzw. hybride Lehrszenarien zu entwickeln.
\end{abstract}

Experiences, Challenges, and Future Teaching Scenarios in Online Teaching. A Mixed-Method-Study on the Covid-19 Summer Semester 2020 from the Perspective of Academics

\begin{abstract}
The summer semester 2020 has challenged academics conducting their courses completely digitally within a very short period of time. How teachers dealt with this, which teaching scenarios they practiced, which potentials and challenges they see in teaching completely
\end{abstract}


remotely, and how they imagine teaching in the future was researched at the Frankfurt University of Applied Sciences using a mixed-method approach with guided interviews and an online questionnaire. In general, academics had a positive overall experience of teaching in the summer semester 2020. They report a great increase in competence, for example, with regard to interaction and communication via video conferencing systems and developing media products. At the beginning of the pandemic, a combination of video conferences, pre-recorded lectures and teaching materials made available via learning platforms were the main foundation of the teaching concept. Central challenges were the lack of a relationship between students and academics and the meaningful design of group work. However, the largely positive experiences of the summer semester 2020 have increased the academics' willingness to offer a combination of traditional face-to-face and online teaching and to develop hybrid teaching scenarios in the future.

\section{Einleitung}

Das SoSe 2020 hat Lehrende durch die Corona-Pandemie unvorbereitet vor grosse Herausforderungen gestellt. Um die Ausbreitung des Virus einzudämmen, mussten an deutschen Hochschulen Präsenzveranstaltungen fast vollständig eingestellt werden. Lehrveranstaltungen wurden an der Frankfurt University of Applied Sciences innerhalb kürzester Zeit auf digital gestützte Formate umgestellt. Bestehende Konzepte, die häufig seit mehreren Jahren etabliert und erprobt waren, bedurften einer kurzfristigen Überarbeitung oder mussten von Grund auf neu konzipiert werden. Auch Lehrende, die digitale Lehrformate bisher nicht genutzt hatten, sahen sich gezwungen die neue Situation anzunehmen und sich mit dem für sie unbekannten Themengebiet auseinanderzusetzen. Zeitgleich wurden an der Hochschule neue technische, wie auch organisatorische Rahmenbedingungen geschaffen, die parallel adaptiert und implementiert werden mussten. Die Lehrenden wurden folglich vor eine massive Umbruchssituation gestellt.

Solche Umbrüche - auch Transitionen genannt - markieren «Veränderungsabschnitte» (Welzer 1993, 36) im Leben eines Menschen, in welchen dieser in einem besonderen Masse gefordert ist, eine neue und unbekannte Lebenssituation zu bewältigen. Transitionen werden auch als «sozial prozessierte, verdichtete und akzelerierte Phasen in einem permanenten Wandel befindlichen Lebenslauf» (Welzer 1993, 37) bezeichnet, in welchen der Alltag von Individuen eine Veränderung erfährt. Für die betroffenen Personen können diese Lebensphasen einerseits sehr lernintensiv und entwicklungsfördernd sein, andererseits bergen sie auch die Gefahr, an den Herausforderungen zu scheitern (Griebel und Niesel 2017). Folglich können Transitionen - abhängig vom Subjekt und der Erfahrung - als mehr oder weniger belastend empfunden werden. 
Übertragen auf die Corona-Pandemie lässt sich folglich festhalten, dass Lehrende vor die Herausforderung gestellt wurden, ihre Lehr- und Lernkonzepte innerhalb sehr kurzer Zeit an die veränderte Situation anzupassen. Anders als im regulären Lehrbetrieb blieb kaum Zeit für die Planung und Gestaltung von reinen Online-Lehrveranstaltungen. Studien zeigen jedoch, dass insbesondere bei der Planung reiner Online-Lehre eine sorgfältige Vorbereitung, die verschiedene Entscheidungsprozesse beinhaltet, wie z. B. die Auswahl der Lernmaterialien, unabdingbar ist (Means, Bakia, und Murphy 2014; Branch und Dousay 2015). Auch Reinmann $(2013,6)$ führt aus, dass insbesondere bei digital gestützter Lehre eine Planung notwendig ist, «allein schon deshalb, weil man z. B. die eingesetzten medialen Inhalte und/oder digitalen Werkzeuge selten ad hoc [...] zur Verfügung stellen kann.» Die Corona-Pandemie fordert Lehrende im SoSe 2020 allerdings heraus, ihre Lehre ohne grosse Vorbereitungsund Planungszeit in ein reines Online-Format umzugestalten. Zudem unterscheidet sich die Situation im SoSe 2020 auch insofern, als dass alle Lehrenden zeitgleich auf digital gestützte Lehr- und Lernangebote umrüsten mussten und diese nicht nur wie sonst üblich - «von einer kleinen Gruppe Lehrender mit besonderen Interessen und Kompetenzen» (Reinmann et al. 2020, 3) umgesetzt werden. Ein weiteres Unterscheidungsmerkmal zwischen regulärer Online-Lehre und der Online-Lehre im Covid-19-Semester 2020 ist, dass üblicherweise Online Veranstaltungen nur dann angeboten werden, wenn dies zu den Zielgruppen, den Lerninhalten, den gewünschten Kompetenzen und den dafür nötigen Rahmenbedingungen sinnvoll und geboten erscheint. Im SoSe 2020 jedoch mussten sämtliche Lehrveranstaltungen (mit sehr wenigen Ausnahmen) komplett auf Online-Lehre umgestellt werden, ohne eine Abwägung der Herausforderungen und Chancen von Online-Lehre durchführen zu können. Unklar ist daher, ob sich Vorteile von E-Learning, wie etwa die Bestimmung und Anpassung an ein individuelles Lerntempo, auch in der aktuellen Situation als diese herauskristallisieren.

Ziel dieser Untersuchung ist es daher herauszufinden, wie Lehrende mit der spezifischen ad-hoc Veränderung umgegangen sind. Entsprechend wurden auf diesen Überlegungen die folgenden vier Forschungsfragen mit dem Fokus auf Veränderungsprozesse in Bezug auf die Lehre entwickelt:

- FF1: Wie erleben Hochschullehrende die Lehrsituation im SoSe 2020 und wie gehen sie mit den Veränderungen um?

- FF2: Wie gestalten sich Lehrszenarien zu Beginn der Pandemie-Situation und wie werden Studierende betreut sowie aktiviert?

- FF3: Welche Potentiale und Herausforderungen sehen Lehrende in reiner OnlineLehre? Welche Massnahmen sehen Lehrende als fördernd für reine Online-Lehre?

- FF4: Wie stellen sich Lehrende zukünftige Lehrszenarien vor? 


\section{Untersuchungsdesign}

Zur Beantwortung der Forschungsfragen wird ein Mixed-Method-Ansatz gewählt. Durch die Verknüpfung von qualitativen Leitfadeninterviews und einem standardisierten Online-Fragebogen soll der Forschungsgegenstand möglichst ganzheitlich untersucht werden. Zum Zeitpunkt der Erhebung gibt es noch keine Befunde über den ad-hoc Transformationsprozess an Hochschulen von Präsenzlehre in OnlineLehre während einer Pandemie. Anders als im regulären Lehrbetrieb blieb keine bzw. kaum Zeit zur sorgfältigen Vorbereitung reiner Online-Lehre, so dass die Handlungsvoraussetzungen anders sind als in vorherigen Semestern. Dies wird auch durch das folgende Zitat untermauert:

«Die Bedingungen, unter denen alle Lehrenden derzeit digitale Lehrangebote produzieren, sind alles andere als ideal und mit den ansonsten üblichen Planungs-, Konzeptions- und Erprobungsabläufen von Online-Lehre nicht vergleichbar» (Reinmann et al. 2020, 2).

Daher erfolgt durch die qualitative Befragung zunächst eine grundlegende explorative Felderkundung, um ein besseres Verständnis für das Phänomen zu generieren (Bryman 2008; Creswell 2003). Aufbauend auf den qualitativen Daten wird dann ein quantitativer Fragebogen entwickelt, dessen Befunde den Geltungsbereich der qualitativen Befunde an der Hochschule prüfen soll (Kuckartz 2014). Ausserdem werden durch den Einsatz beider Methoden «blinde Flecken» oder Erklärungslücken» (Kelle 2014, 162) ausgeglichen. In den nachfolgenden Abschnitten werden zunächst die Erhebungsinstrumente vorgestellt, bevor dann auf die Durchführung des Pre-Tests sowie auf die Stichprobe und die Auswertungsstrategie eingegangen wird.

\subsection{Methoden: Qualitative Leitfadeninterviews und standardisierte Online-Befragung} Ein qualitatives Vorgehen bietet sich speziell dann an, wenn - wie in diesem Fall noch keine einschlägigen theoretischen Erkenntnisse zu einem Forschungsgegenstand vorliegen (Lamnek 2010). In der qualitativen Forschung wird meist auf Interviewtechniken zurückgegriffen, da diese den Befragten die Möglichkeit geben, über «ihre Biographie, Weltsicht, Erfahrungen und Kontexte zu berichten» (Friebertshäuser 1997, 371). Forschende erhalten auf diese Weise einen direkten Zugang zu den betroffenen Personen und Forschungsbereichen und es kann damit «eine von der Forscherin wahrgenommene gesellschaftliche Problemstellung, mit der die Befragten umzugehen haben» (Friebertshäuser und Langer 2010, 442) untersucht werden. D. h. mittels eines Interviews kann ein Einblick in die subjektive Sichtweise einer Person generiert werden, was insbesondere in dieser Studie in einem ersten Schritt von Bedeutung ist. Denn so kann zunächst herausgefunden werden, welche individuellen Strategien Lehrende entwickelt haben, um auf die ad-hoc Umstellung zu reagieren und welche Vor- und Nachteile sie in reiner Online-Lehre sehen. 
In dieser Studie kommen speziell Leitfadeninterviews zum Einsatz, da sich die Methode durch eine offene Atmosphäre auszeichnet, die ein flexibles Vorgehen vorsieht. Zudem bietet sie die Möglichkeit, vertiefende Fragen zu stellen, um Missverständnisse durch gezielte Nachfragen aus dem Weg zu räumen (Flick 2011). Leitfadeninterviews haben ferner den Vorteil, dass Ergebnisse besser miteinander verglichen werden können. Bei der Gestaltung von ebensolchen wird empfohlen, zunächst allgemeine Fragen zu stellen, die dann sukzessive von detaillierten Fragestellungen abgelöst werden (Meyen et al. 2011). Ausserdem sollten die Fragen die Interviewpartnerin bzw. den Interviewpartner zum Nachdenken anregen, sich auf deren bzw. dessen Erfahrungen beziehen, die Antwort nicht suggerieren und leicht verständlich sein (Meyen et al. 2011). Auch wenn Leitfadeninterviews Vorteile wie z. B. Flexibilität oder Offenheit bieten, hat die Methode auch Nachteile, was im Abschnitt Limitationen vertiefend erläutert wird. Nachstehend wird nun auf die Konzeption des Leitfadens eingegangen. Dabei dienten die eben vorgestellten Empfehlungen als Grundlage für die Leitfadengestaltung.

Der Leitfaden dieser Studie besteht aus insgesamt vier Themenkomplexen, die der Beantwortung der Forschungsfragen dienen sollen. Dabei werden - wie von Meyen et al. (2011) empfohlen - zunächst allgemeine Fragen gestellt, die sich dann ins Spezielle bewegen.

Der erste Themenkomplex beschäftigt sich mit dem Erleben und den Veränderungen der Lehre, der Prüfungen und sonstiger Rahmenbedingungen im SoSe 2020. Dieser Themenblock dient zur Beantwortung von FF1 und FF2. Zum Einstieg in das Gespräch werden die Lehrenden gebeten zu erzählen, wie sie sich in das SoSe 2020 eingefunden haben. Anschliessend wird gefragt, was sich seit Semesterbeginn im Vergleich zu früheren Semestern konkret in Bezug auf die Planung und Umsetzung der Lehrveranstaltung verändert hat. Dabei wird den Lehrenden zusätzlich die Möglichkeit gegeben, beispielhaft zu berichten, wie eine Lehrveranstaltung in der Pandemie-Situation abläuft. Ausserdem soll mit der Frage «Wie sind Sie mit der Veränderung umgegangen?» herausgefunden werden, welche Bewältigungsstrategien die Befragten entwickelt haben, um mit diesem Umbruch umzugehen.

Mit dem zweiten Themenkomplex wird ermittelt, ob Lehrende neue Lehrformen praktizieren und wo sie Potentiale sowie Herausforderungen reiner Online-Lehre sehen. Dieser Themenkomplex soll bei der Beantwortung von FF2 und FF3 helfen. Dazu werden zunächst Fragen wie «Praktizieren Sie eine neue Lehrform? Wenn ja, was ist anders?», «Welche Lernmaterialien stellen Sie den Studierenden zur Verfügung?» und «Hat die Gestaltung dieser Lehrform einen Mehraufwand für Sie bedeutet? Wenn ja, wie gross war dieser?» gestellt. Anschliessend werden die Probandinnen und Probanden nach den Potentialen und Herausforderungen reiner Online-Lehre gefragt: «Wo sehen Sie Potentiale reiner Online-Lehre?», «Wo liegen die Herausforderungen 
reiner Online-Lehre?», "Gibt es zwischen den Lehrveranstaltungstypen Unterschiede in Hinblick auf die Herausforderungen und wenn ja welche?» und «Gibt es Herausforderungen, die Sie bislang nicht lösen konnten? Wenn ja, welche?».

Im dritten Themenkomplex wird konkret erforscht, wie Studierende von Lehrenden betreut und aktiviert werden. Mit diesem Themenkomplex soll ebenfalls FF2 beantwortet werden. Dazu werden Fragen wie «Wie betreuen Sie Studierende aktuell?» und «Wie haben Sie in den Lehrveranstaltungen Aktivitäten zwischen den Studierenden gefördert?» gestellt.

Mit dem letzten Themenabschnitt wird erforscht, welche Lehrszenarien sich Lehrende in Zukunft vorstellen können. Dieser Themenkomplex beinhaltet Fragen wie "Haben Sie bereits eine Vorstellung, wie Sie Ihre Lehre in Zukunft gestalten möchten?» und «Welche Szenarien können Sie sich langfristig vorstellen?» (FF4). Das Interview endet mit der Frage «Gibt es noch etwas, was Sie gerne ergänzen würden?». Damit wird den Probandinnen und Probanden die Möglichkeit eingeräumt, Aspekte zu nennen, die bis dahin eventuell noch nicht beleuchtet wurden.

Aufbauend auf den Befunden der qualitativen Studie wurde ein standardisierter Online-Fragebogen mit offenen und geschlossenen Fragen - ähnlich wie bei Reinmann et al. 2020 - erstellt. Ziel war es, wie eingangs erläutert, mittels des standardisierten Fragebogens die Befunde der qualitativen Studie zu prüfen (Kuckartz 2014). Der Fragebogen ist inhaltlich in verschiedene Themenblöcke unterteilt.

Nach einer Einleitung, die den Zweck des Fragebogens erläutert, folgt der erste inhaltliche Abschnitt, in dem allgemeine Fragen zu Status der Lehrenden, Fachbereichszuordnung, Lehrerfahrung an Hochschulen sowie den im SoSe 2020 angebotenen Veranstaltungsarten und der Teilnahme durch Studierende gestellt werden. In diesem Abschnitt werden neben der Anzahl der geplanten und durchgeführten Veranstaltungen auch Fragen danach gestellt, ob sich die Prüfungsform geändert hat und ob elektronische Prüfungen auch in Zukunft als Prüfungsinstrument für die Befragten vorstellbar sind. Diese Fragen dienen einerseits der Beantwortung von FF1 und andererseits auch der Einordnung der Rückläufe - so kann überprüft werden, ob alle Fachbereiche, Veranstaltungsformen und Statusgruppen in der Befragung abgebildet sind.

Die Planung und Gestaltung digital gestützten Lehrens sind Gegenstand des zweiten Abschnitts des Fragebogens. Dabei werden Begriffe erfragt, die die Lehrenden mit der Online-Lehre im SoSe 2020 verbinden - hier wurde für die Auswahl der möglichen Begriffe insbesondere auf die Ergebnisse der qualitativen Interviews zurückgegriffen. In diesen hatten sich verschiedene Assoziationen, wie z. B. «örtlich unabhängig», «aufwändig» und "anonym», herauskristallisiert. Weiterhin wird die von den Befragten selbst wahrgenommene digitale Kompetenz (z. B. Interaktion und Kommunikation über Videokonferenzsysteme oder Erstellen von Medienprodukten) aktuell und im Rückblick vor dem SoSe 2020 abgefragt. Diese Fragenblöcke sollen 
insbesondere dabei helfen, FF1 zu beantworten. Weitere Fragen betreffen den Aufwand der Online-Lehre im SoSe 2020 sowie den erwarteten Aufwand für möglicherweise weitere kommende Online-Semester.

Ein weiterer Themenblock beinhaltet Fragen zur Interaktion, Kommunikation und Betreuung von Studierenden, zur digitalen Lernumgebung sowie den grundsätzlichen Potentialen und Herausforderungen, welche die Befragten bei digital gestütztem Lehren und Lernen sehen. In diesem Kontext wurden den Lehrenden Fragen gestellt wie etwa «Wie haben Sie die Zusammenarbeit der Studierenden untereinander im SoSe 2020 gefördert? (Mehrfachnennung möglich)» (Antwortmöglichkeiten u. a.: Bereitstellung eines Leitfadens für die Gruppenarbeit und Bereitstellung von Tools für die gemeinsame Arbeit). Weiterhin werden in diesem Abschnitt Lehrende zu möglichen zukünftigen Lehrszenarien befragt (z. B. «Wie planen Sie in Zukunft E-Learning überwiegend einzusetzen?»; Antwortmöglichkeiten u. a.: Ich möchte mit E-Learning eine Kombination aus Präsenz- und Onlinelehre konzipieren (z. B. Blended-LearningFormate und Ich möchte mit E-Learning reine Online-Lehrveranstaltungen konzipieren). Er dient insbesondere der Beantwortung von FF2, FF3 und FF4. Es werden den Lehrenden Fragen zur Relevanz von synchroner und asynchroner sowie persönlicher Kommunikation mit den Studierenden gestellt. Ferner wird danach gefragt, welchen Studierenden (Bachelor-, Master-, Dual-Studierenden sowie Studierenden in niedrigen oder hohen Fachsemestern) es nach Eindruck der Lehrenden am leichtesten fiel, an den Online-Veranstaltungen teilzunehmen. Zudem gibt es einige Fragen zu Herausforderungen, welche die Lehrenden konkret bewältigen mussten, sowie Freitexte zur Beantwortung der Frage, wie diese gemeistert wurden. Dazu werden verschiedene Lehr- und Lernformen und technische Systeme abgefragt, welche die Lehrenden im SoSe 2020 eingesetzt haben. Darüber hinaus wird mit Bezug zu verschiedenen Zielen der Lehre (etwa: Anwendungs- und Praxisbezüge herstellen oder die Studierenden aktivieren) gefragt, inwiefern die Lehrenden sich in der Lage sahen, diese in der Online-Lehre umzusetzen. Weiterhin gibt es Fragen dazu, ob die Probandinnen und Probanden digital gestützte Lehre grundsätzlich als eher nachteilig oder eher vorteilhaft für bestimmte Aspekte der Lehre (etwa: Flexibilisierung, persönliche Betreuung oder kollaboratives Arbeiten) einschätzen. Auch diese wurden überwiegend aus den qualitativen Interviews abgeleitet. Den Abschluss des Abschnitts und des Fragebogens bilden einige zukunftsgerichtete Fragen. Diese betreffen etwa die Bereitschaft auch in Zukunft überwiegend Online-Lehre einzusetzen oder bestimmte digitale Tools, Lernmaterialien oder Methoden dauerhaft in die Lehre zu integrieren. 


\subsection{Pretest}

Anfang Mai 2020 wurde zunächst ein Pretest mit zwei Lehrenden durchgeführt, um den Aufbau und die Struktur des Leitfadens zu testen. Die Probeinterviews zielten ausserdem darauf ab, die inhaltliche Konzeption des Leitfadens zu prüfen und eine gewisse Routine im Hinblick auf den Ablauf des Gesprächs zu entwickeln. Für die inhaltliche Konzeption der Leitfadeninterviews wurden nach Durchführung des Pretests nochmals die Anordnung der Fragen und vereinzelt das Wording von Fragen angepasst. Der aus den qualitativen Daten entwickelte Fragebogen wurde zwei Lehrenden geschickt mit der Bitte zu prüfen, ob die Fragen verständlich und nachvollziehbar sind. Begriffe wie z. B. Scannerklausur oder Proctoring wurden u. a. nochmals definiert, da sie den Lehrenden nicht bekannt waren.

\subsection{Stichprobe und Erhebungszeitraum}

Für die qualitative Teilstudie wurden insgesamt elf Lehrende der Hochschule befragt, die sich in vier Interviews mit Frauen und sieben Interviews mit Männern aufteilen. Es sind alle Fachbereiche, das Fachsprachenzentrum sowie das Interdisziplinäre Studium Generale vertreten. Bei den befragten Personen handelt es sich um Professorinnen und Professoren sowie Lehrpersonen für besondere Aufgaben. Es wurden auch Lehrbeauftragte angefragt, die sich allerdings nicht zurückgemeldet haben. Die Probandinnen und Probanden wurden im Mai und Juni 2020 nach deren Zustimmung über ein Videokonferenzsystem befragt.

Der Befragungszeitraum des quantitativen Fragebogens lief von Ende August bis Mitte Oktober 2020. Angeschrieben wurden alle Lehrenden der Frankfurt University of Applied Sciences (Professorinnen und Professoren, Mitarbeitende mit Lehrdeputat, Lehrbeauftragte, Lehrpersonen für besondere Aufgaben) - insgesamt 1140 Personen. Der Fragebogen wurde von 242 Personen ausgefüllt, was einer Rücklaufquote von $21,2 \%$ entspricht. Bei den Statusgruppen stellen die Professorinnen und Professoren mit 46,6\% die grösste Gruppe der Befragten, gefolgt von Lehrbeauftragten mit 36,6\%, während 10,1\% Mitarbeitende mit Lehrdeputat und 5,5\% Lehrpersonen für besondere Aufgaben sind ( $n=238)$. Der Grossteil der Befragten $(47,3 \%)$ ist schon neun Jahre oder länger in der Hochschullehre tätig, 17,3\% sind seit mindestens zwei Jahren in der Lehre tätig, 14,3\% geben an seit fünf bis sechs Jahren zu lehren und 11,8 \% lehren seit drei bis vier Jahren $(n=237)$. Alle vier Fachbereiche sind bei der Befragung vertreten ebenso wie das Interdisziplinäre Studium Generale und das Fachsprachenzentrum (Fb 1 18,8\%, Fb 2 28\%, Fb 3 20,9\%, Fb 4 25,1\%, FSZ 6,7\%, !SG 4,2\%, n=239). Alter und Geschlecht der Befragten wurden aus Datenschutzgründen nicht erhoben, da sonst ein Rückschluss auf einzelne Personen möglich gewesen wäre. 


\subsection{Auswertungsstrategien}

Die qualitativen Daten wurden zunächst transkribiert. Bei den Transkripten wurde sich überwiegend an die Transkriptionsregeln von Dresing und Pehl (2015) gehalten. Anschliessend wurden die Daten mittels des thematischen Codierens von einer Codiererin in zwei Codiervorgängen nach Hopf und Flick in MAXQDA ausgewertet. Die Kategorien wurden induktiv aus dem Datenmaterial gebildet. Beispielhaft wird nachfolgend ein Ausschnitt der Kategorie «Potentiale reiner Online-Lehre» inklusive der Subcodes dargestellt:

\section{Potentiale}

- Organisation von Meetings

- Durchführung von Online-Sprechstunden

- Direkte Ansprache von Studierenden in VK-System

- Mehr Zeit für Fragen

- Flexibilität

- Bildschirmfreigabe zur gemeinsamen Besprechung

- Förderung des Selbststudiums und individuelles Lerntempo

- Wiederholung von Lerninhalten

Die Daten des standardisierten Online-Fragebogens wurden zunächst mittels EvaSys erhoben und anschliessend mit Methoden der deskriptiven Statistik ausgewertet. Zum Einsatz kamen insbesondere Häufigkeitsauszählungen und beim Vergleich der Statusgruppen und Fachbereiche ein Mittelwertvergleich. Die Auswertung der Daten, wie etwa der Mittelwertvergleich und die Häufigkeitsauszählung, erfolgte direkt durch EvaSys. Mit dem Instrument zur Messung des Lernerfolgs nach Raupach et al. (2011) wurde ebenfalls in EvaSys ausgewertet, wie Lehrende ihre digitalen Kompetenzen (z. B. Erstellung von Medienprodukten) vor und nach dem Covid19-Semester einschätzen. Dabei werden die Befragten gebeten ihre digitalen Kompetenzen aktuell und rückblickend vor Beginn des Covid-19-Semesters zu bewerten. Nach Raupach und Schiekirka (o. J.) ist eine punktuelle Selbsteinschätzung aufgrund einer Tendenz zur Über- oder Unterschätzung fraglich hinsichtlich der Validität, eine wiederholte oder rückblickende Selbsteinschätzung jedoch vermeidet dieses Problem, da die Fähigkeit zur Selbsteinschätzung weitgehend stabil sei. Die Reliabilität und Validität dieser Form der Lernerfolgsevaluation wurde bereits in einigen Studien der medizinischen Lehrforschung untersucht (Raupach und Schiekirka o. J.).

Die offenen Fragen des standardisierten Fragebogens wurden ebenfalls mittels des thematischen Codierens in MAXQDA ausgewertet. 


\section{Befunde der qualitativen Teilstudie}

Die Befunde der qualitativen Interviews zeigen, dass die Umstellung auf reine OnlineLehre zu vielfältigen Veränderungen $(F F 1)$, wie z. B. veränderten Prüfungsformen, einem verstärkten Einsatz des Lernmanagementsystems Moodle und einer erhöhten Arbeitslast führte. Insgesamt wurde das SoSe 2020 von den Befragten aber positiv wahrgenommen. Zwei Lehrende heben die Möglichkeit, sich trotz erheblichen Mehraufwands im Bereich E-Learning weiterbilden zu können, als sehr bereichernd und entwicklungsfördernd hervor, wie dieses Zitat untermauert:

«Auch wenn Corona etwas Furchtbares ist. Aber, dass wir jetzt verpflichtet waren, wir haben uns alle vorgenommen: Ja, E-Learning, das machen wir auf jeden Fall, aber nächstes Semester. Jetzt waren wir einfach verpflichtet es zu machen. Und ich habe das Gefühl, dass ich viel gelernt habe. Insofern ist dieser Zusatzaufwand - nehme ich niemandem übel. Im Gegenteil, ich bin fast dankbar, dass ich diesen Kick bekommen habe» (Interview 5).

In Bezug auf $F F 2$ haben sich fünf verschiedene Lehrszenarien herauskristallisiert, wie die nachfolgende Tabelle darstellt.

\begin{tabular}{|l|l|l|}
\hline Lehrszenario & Lernform & Beschreibung \\
\hline $\begin{array}{l}\text { Vortragsbasierte } \\
\text { Vorlesung mit Skript } \\
\text { naleodle } \text { Aufzeichnung }\end{array}$ & $\begin{array}{l}\text { überwiegend } \\
\text { synchron }\end{array}$ & $\begin{array}{l}\text { Die Lehrveranstaltung findet live über ein Videokon- } \\
\text { ferenzsystem - ähnlich wie der Präsenztermin - statt. } \\
\text { In Moodle werden Skripte inkl. Literaturhinweisen zur } \\
\text { Verfügung gestellt. Teils werden die Live-Lehrveranstal- } \\
\text { tungen zusätzlich aufgezeichnet und den Studierenden } \\
\text { bereitgestellt. Dieses Lehrszenario zeichnet sich durch } \\
\text { einen frontalen Vortrag mit wenig Interaktion aus. }\end{array}$ \\
\hline $\begin{array}{l}\text { Diskursive Vorlesung } \\
\text { mit Gruppenarbeit in } \\
\text { Breakout-Rooms }\end{array}$ & $\begin{array}{l}\text { überwiegend } \\
\text { synchron }\end{array}$ & $\begin{array}{l}\text { Die Lehrveranstaltung findet live statt. Die Studierenden } \\
\text { werden während der Vorlesung regelmässig in Breakout- } \\
\text { Rooms geschickt, um gemeinsam Aufgaben zu bearbei- } \\
\text { ten. }\end{array}$ \\
\hline $\begin{array}{l}\text { Diskursives Seminar } \\
\text { mit Gruppenarbeit in } \\
\text { Breakout-Rooms }\end{array}$ & $\begin{array}{l}\text { überwiegend } \\
\text { synchron }\end{array}$ & $\begin{array}{l}\text { Diese Lehrveranstaltung lebt insbesondere von Gruppen- } \\
\text { arbeit. Es wird gemeinsam über das Semester synchron } \\
\text { in einer Videokonferenz an einer Aufgabenstellung oder } \\
\text { einem Projekt gearbeitet bzw. wird gemeinsam die Be- } \\
\text { nutzung von Fach-Software eingeübt. }\end{array}$ \\
\hline $\begin{array}{l}\text { Diskursives Semi- } \\
\text { nar/Vorlesung mit } \\
\text { wöchentlichen } \\
\text { Arbeitspaketen und } \\
\text { synchroner Bespre- } \\
\text { chung }\end{array}$ & $\begin{array}{l}\text { synchron und } \\
\text { asynchron }\end{array}$ & $\begin{array}{l}\text { Studierende erhalten einen Lehrplan mit wöchentlichen } \\
\text { Aufgaben, die asynchron bearbeitet werden. Sie müssen } \\
\text { teils zusätzlich Fragen formulieren. In der Videokonfe- } \\
\text { renz werden im Anschluss gemeinsam die Fragen und } \\
\text { Aufgaben besprochen. }\end{array}$ \\
\hline
\end{tabular}




\begin{tabular}{|c|c|c|}
\hline Lehrszenario & Lernform & Beschreibung \\
\hline $\begin{array}{l}\text { Diskursive Lehrver- } \\
\text { anstaltung mit wö- } \\
\text { chentlichen Arbeits- } \\
\text { paketen, Lernfilmen/ } \\
\text { VL-Aufzeichnungen } \\
\text { und synchroner Be- } \\
\text { sprechung }\end{array}$ & $\begin{array}{l}\text { synchron und } \\
\text { asynchron }\end{array}$ & $\begin{array}{l}\text { Es wird ein Zeitplan in Moodle zur Verfügung gestellt, der } \\
\text { Studierenden eine Orientierung bietet, wann welches } \\
\text { Thema bearbeitet werden soll. In einem Moodle-Kurs } \\
\text { werden Vorlesungsaufzeichnungen und Folien mit Fra- } \\
\text { gen zur Beantwortung zum Thema bereitgestellt. Diese } \\
\text { sollen von den Studierenden bearbeitet werden. Die } \\
\text { Lösungen zu den einzelnen Fragen werden nicht online } \\
\text { gestellt, sondern in einer Zoom-Konferenz besprochen. } \\
\text { Die einzelnen Bereiche werden nach und nach in Moodle } \\
\text { freigeschaltet. }\end{array}$ \\
\hline
\end{tabular}

Tab. 1.: Zusammenfassung der beschriebenen Lehrszenarien zu Beginn der Pandemie.

Die Betreuung der Studierenden (FF2) fand im SoSe 2020 über verschiedene Kommunikationskanäle statt. Am häufigsten werden Online-Sprechstunden via Zoom $(n=6)$ und E-Mails $(n=5)$ genannt. Zum Einsatz kam ferner das Forum in Moodle $(n=4)$. Die Lehrenden berichten von einer Aktivierung überwiegend über die sogenannten Breakout-Rooms:

"In einer Veranstaltung habe ich, indem ich die Breakout Sessions genutzt habe. Da hatten wir eine etwas komplexere Aufgabe und dann habe ich in Kleingruppen, also sie mussten es in Kleingruppen bearbeiten. Ich hatte zu der Veranstaltung auch einen Gastdozenten. Sie konnten ihn dann immer fragen, wenn sie Probleme hatten und er ist dann in die einzelnen Sessions gegangen» (Interview 4).

Vereinzelt berichten Lehrende aber auch, dass die Aktivierung für sie eine der grössten Herausforderungen darstellt, die sie bislang noch nicht befriedigend lösen konnten. Auf weitere Herausforderungen und Potentiale wird in den nachfolgenden Abschnitten vertieft eingegangen.

Auf die Frage, wo Lehrende Potentiale reiner Online-Lehre sehen (FF3), hat sich für einen Grossteil der Lehrenden ( $n=7)$ die Möglichkeit des Selbststudiums als besonders wertvoll herausgestellt. Da auf diese Weise die verschiedenen Lernstiltypen bedient werden können, wie die beiden nachfolgenden Zitate untermauern:

«Ich glaube, es ist ein effizienteres Lernen, eine effizientere Art des Lernens. Dadurch, dass jeder in seinem eigenen Tempo lernen kann» (Interview 4).

«Ja, gerade darin, dass sie durch [...] das Selbststudium, zu welchem Zeitpunkt man auch will. Diese Veranstaltung kann man viel effizienter lernen, also wirklich viel selbstbestimmter» (Interview 6). 
Auch wird die Möglichkeit des zeitunabhängigen Lernens positiv bewertet ( $n=6)$. In Bezug auf die Konzeption von reinen Online-Lehrveranstaltungen wird zudem berichtet, dass diese nun sehr viel strukturierter sind, da vorab verstärkt reflektiert werden muss, wie Kurse didaktisch konzipiert werden. Ferner wird die Möglichkeit der Bildschirmfreigabe zur gemeinsamen Besprechung von Lerninhalten und Aufgaben $(n=5)$ als ein weiterer Vorteil genannt. Als förderlich für die Gestaltung von OnlineLehrveranstaltungen werden von den Lehrenden in den Interviews folgende Aspekte genannt:

- Aufbau von Vertrauen zwischen Lehrenden und Studierenden und zwischen Studierenden untereinander;

- Schnelle Kommunikation zwischen Lehrenden und Studierenden;

- Bildung von Arbeitsgruppen mit Koordinatorinnen bzw. Koordinatoren, welche Fragen in ihren Gruppen sammeln und an die dozierende Person weiterleiten;

- Strukturierter Ablauf mit wöchentlichen Arbeitspaketen, die sukzessive freigeschaltet und gemeinsam besprochen werden;

- Zurverfügungstellung von Lernmaterialien für das Selbststudium mit anschliessender Besprechung;

- Zwischentests zur Lernstandskontrolle.

Auch wenn Lehrende Potentiale in der Online-Lehre sehen, nennen sie auch Herausforderungen, die sie mitunter noch nicht lösen konnten. Als grösste soziale Herausforderung wird die Atmosphäre bzw. fehlende Interaktivität in Videokonferenzen genannt, da die Studierenden häufig ihre Kameras und Mikrofone ausgeschaltet lassen $(n=9)$ und dadurch keine Beziehung aufgebaut werden kann:

«Aber für mich ist Hochschule und Bildung vor allem auch eine Beziehungsgeschichte. Das fällt bei einem flächendeckend online zu viel weg. Man hat keine Beziehung zu den Studenten mehr» (Interview 2).

«Und dann sehe ich nur so schwarze Kästchen. Und dann redet man anderthalb Stunden gegen so schwarze Kästchen. Das ist irgendwie wenig angenehm» (Interview 11).

Auch fehlen Lehrenden die Ad-Hoc-Gespräche, die häufig vor bzw. nach einer Lehrveranstaltung in Form von Small-Talk stattfinden. Insbesondere bei Videokonferenzen entsteht durch die genannten Faktoren eine Lernatmosphäre, die sich durch Passivität auszeichnet. Als eine weitere Herausforderung wird die Beziehung der Studierenden untereinander genannt. Kennen sich diese bereits aus vorherigen Semestern, ist die Zusammenarbeit meist kein Problem. Sind sich die Studierenden allerdings noch nicht vertraut, so gestaltet sich die Kollaboration teils schwierig:

«Es war, wenn sie sich nicht kennen, sehr, sehr schwierig» (Interview 7). 
In Bezug auf organisatorische Herausforderungen wird von verschiedenen Lehrenden berichtet, dass sich nicht alle Lerninhalte/Lernformen, wie z. B. BaustellenExkursionen, Rundgänge, Sprachkurse, Reflexionen, PC-Pool-Übungen oder Laborsituationen rein digital vermittelt lassen:

«Und das habe ich gestrichen, oder auch einen virtuellen Rundgang. Hatten wir eigentlich einen echten Rundgang [...] vor» (Interview 3 ).

«Und was bei uns eine Herausforderung wirklich war, waren die PC Pools, die PC Pools abzubilden. Das war echt eine Riesenherausforderung [...]. Veranstaltungen, das heißt [...] für die Lehre in den PC mit unterschiedlichen Konfigurationen» (Interview 10).

Als eine weitere Einschränkung werden grosse Gruppen genannt, da es bei diesen schwieriger ist, eine interaktive Lernumgebung zu gestalten:

«Wenn ich eine Gruppe von 40 in Gruppen einteilen muss, geht das, aber wenn ich eine Gruppe von 200 in Gruppen einteilen muss, von denen ich gar nicht weiss, welche überhaupt erst mit-machen, ist das irgendwie chancenlos» (Interview 6).

In den Interviews herrscht Konsens darüber, dass reine Online-Lehre keine Option für zukünftige Lehrszenarien ist (FF4). Der überwiegende Teil der Lehrenden möchte wieder in die Präsenzlehre zurückkehren und E-Learning dort einsetzen, wo es ihrer Meinung nach Sinn macht. Insbesondere bei der Vermittlung von Grundlagenwissen möchten einige Lehrende verstärkt Lernvideos anbieten (FF4). Nachdem die qualitativen Befunde dargestellt wurden, folgt die Darstellung der Befunde des standardisierten Online-Fragebogens.

\section{Befunde quantitative Teilstudie}

Was das Erleben der Lehrsituation im Online-Semester 2020 angeht (FF1), bestätigen sich in der quantitativen Befragung die Eindrücke der Interviews: Durch die Umstellung auf Online-Lehre hat sich die Arbeitslast bei mehr als der Hälfte der befragten Personen mindestens verdoppelt $(n=236)^{1}$. Wie die Lehrenden das SoSe 2020 erlebt haben, lässt sich auch anhand deren Assoziation mit bestimmten Begriffen zeigen. Auf Basis der Ergebnisse der qualitativen Teilstudie wurden die Befragten gebeten, die drei Begriffe auszuwählen, die sie am meisten mit dem Erleben des Online-Semesters in Verbindung bringen. Am häufigsten wurden dabei die Begriffe «örtlich unabhängig» $(44,8 \%)$, «aufwändig» $(41,4 \%)$ sowie «distanziert» $(36,4 \%)$ genannt $(n=239)$. Am seltensten hingegen wurden die Begriffe «nah» $(4,2 \%)$, «bessere Organisation» (10\%) und «einseitig» (11,7\%) ausgewählt.

1 Das $n$ gibt jeweils die Gesamtzahl an Antworten an. 


\section{Was hat für Sie digital gestütztes Lehren und Lernen im SoSe} 2020 ausgemacht? ( $n=239)$

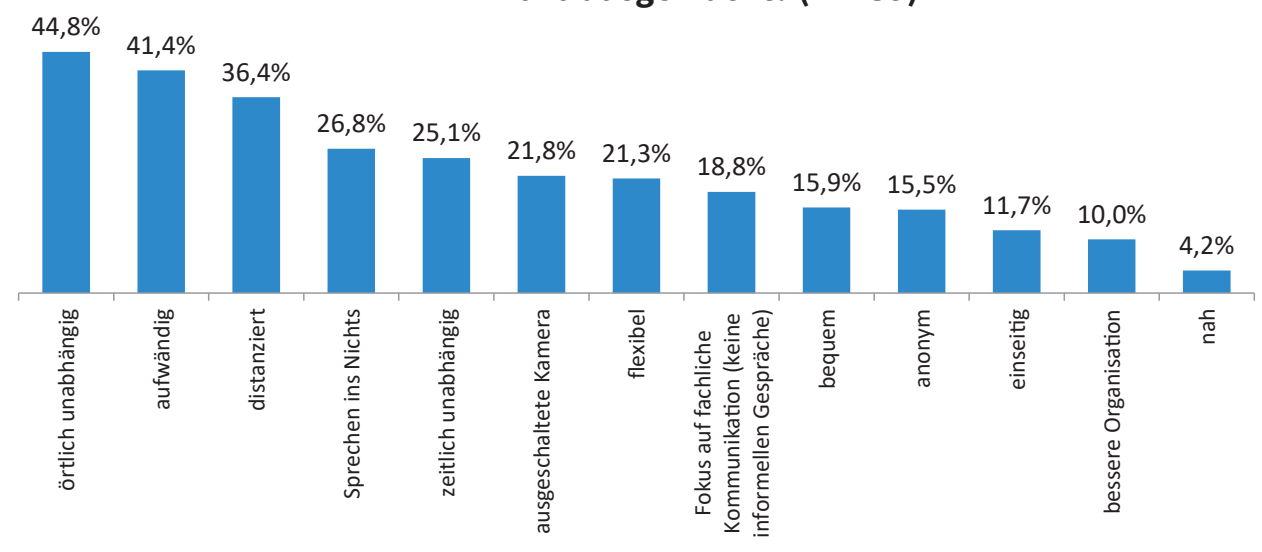

Abb. 1.: Assoziationen mit dem SoSe 2020.

In Bezug auf $F F 1$ geben 25,5\% der Befragten an, die Prüfungsform in ihrer Lehrveranstaltung bedingt durch das Covid-19-Semester geändert zu haben $(n=235)$. In der dazugehörigen offenen Frage führen einige Personen aus, dass sie z. B. mündliche Prüfungen in schriftliche Abgaben umgewandelt haben $(n=6)$. Zudem wurden Präsenzprüfungen in Online-Formate umgewandelt. So geben 14 Personen an, dass sie mündliche Prüfungen über ein Videokonferenzsystem abgehalten haben. Was die Lehrveranstaltungsarten angeht, gibt mehr als die Hälfte der Lehrenden an $(59,4 \%)$, dass sie im SoSe 2020 Vorlesungen angeboten haben, etwas weniger als die Hälfte gibt jeweils an, Übungen und Seminare durchgeführt zu haben $(n=239)$. An den klassischen Formaten wurden also im Gegensatz zur Prüfungsform festgehalten. Was den Aufwand der Online-Lehrveranstaltungen angeht, geben über die Hälfte der Befragten $(54,2 \%)$ an, dass dieser im SoSe 2020 doppelt so hoch gewesen sei wie in Nicht-Corona-Semestern ( $n=236)$. In den offenen Antworten wird deutlich, dass sich insbesondere Lehrbeauftragte eine monetäre Anerkennung der erheblichen Mehraufwände gewünscht hätten.

Die Notwendigkeit sich ad-hoc mit Online-Lehre zu beschäftigen, führte bei vielen Lehrenden auch zu einem wahrgenommenen Kompetenzzuwachs. So sehen bei einer rückblickenden Betrachtung der digitalen Kompetenzen vor und nach dem SoSe 2020 die Befragten eine deutliche Veränderung. Analog zum Lernerfolg einer Lehrveranstaltung kann von einem Lernerfolg von $50,5 \%$ gesprochen werden $(n=241)$. 


\section{Lernerfolgsevaluation}

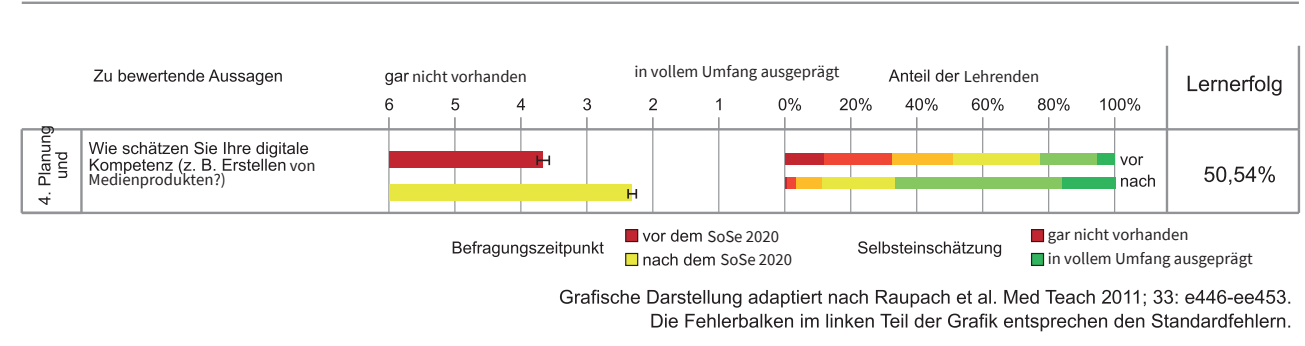

Abb. 2.: Wahrgenommener Kompetenzzuwachs im Bereich digitale Kompetenzen.

Die Bewältigung der Herausforderungen wird von einigen Lehrenden als positiv erlebt. So nennen als Antwort auf die offene Frage «Was ist Ihnen aus Ihrer subjektiven Sicht im SoSe 2020 besonders gelungen im Hinblick auf Ihre Lehrveranstaltungen?» 34 Lehrende die Entwicklung von neuem Lehrmaterial und 21 Personen haben die Kommunikation und Interaktion mit Studierenden als besonders gelungen erlebt.

In Hinsicht auf FF2 hat sich herauskristallisiert, dass synchrone Vorträge via eines Videokonferenzsystems mit 76,9\% die am häufigsten eingesetzte Lehrform sind, gefolgt von asynchronen Formaten mit aufgezeichneten Inputs wie z. B. Vorträge $(49,6 \%)(n=239)$. Dabei gibt über die Hälfte $(57,1 \%)$ der Lehrenden an, dass synchrone Kommunikation für ihre Lehrveranstaltung sehr relevant sei, 30\% geben an, dass sie eher relevant sei $(n=233)$. Einige Lehrende führen in der offenen Frage aus, dass sie in synchronen Settings zudem Diskussionen mit Studierenden geführt haben $(n=16)$ und regelmässig Audience-Response-Systeme bzw. das Umfrage-Tool des Videokonferenzsystems verwendet haben $(n=8)$, um die Studierenden zu aktivieren. In asynchronen Settings wurde insbesondere auf die Bearbeitung von Aufgaben $(n=14)$ und Lernstandskontrollen via Moodle gesetzt ( $n=12$ ), um die Aktivierung zu fördern.

Sofern eine Zusammenarbeit zwischen Studierenden vorgesehen war, wurde diese am häufigsten (65,3\%) mit einer gemeinsamen Aufgabenstellung umgesetzt $(n=239)$. In den offenen Fragen wurde dies von 14 Personen teils noch expliziert durch Aussagen wie «asynchrone Bearbeitung von Fallstudien mit regelmässigem und gruppenindividuellen Feedback» oder «Nach jeder Erklärung müssen die Studierenden Übungen lösen, die auf der Erklärung basieren. Viele dieser Übungen sind in Gruppen.»In Bezug auf die Betreuung der Studierenden hat sich gezeigt, dass Lehrende am häufigsten $(95,0 \%)$ via E-Mail mit ihren Studierenden kommunizieren, gefolgt von individuellen virtuellen Sprechstunden $(81,2 \%)(n=239)$.

Als vorteilhaft bzw. fördernd wurde die Möglichkeit des zeit- und ortsunabhängigen Lernens $(96,6 \%)$, die Wiederholbarkeit der Lerninhalte $(88,2 \%)$ sowie die Wiederverwertung der Lernmaterialien $(87,1 \%)$ genannt $(n=204)(F F 3)$. Am wenigsten vorteilhaft schätzten Lehrende digital gestütztes Lehren und Lernen für informelle 
Kommunikation (83,7\%), Aktivierung der Studierenden $(77,3 \%)$ und kollaboratives Arbeiten der Studierenden (76,7\%) ein ( $n=204)$. Hier können die Befunde der qualitativen Interviews bestätigt werden.

Dementsprechend identifizieren die Lehrenden als die grössten Herausforderungen $(F F 3)$, denen sie mit ihren Studierenden gegenüberstanden, nicht-aktivierte Kameras $(60,3 \%)$, fehlende Beteiligung der Studierenden und deren fehlende Beziehung untereinander (jeweils 53,6\%) ( $n=239)$. Auf die offene Frage «Wie können diese Herausforderungen überwunden werden?» antworten die Lehrenden u. a., dass sie verstärkt aktivierende Methoden, wie z. B. Polls einsetzen möchten und einen stärkeren Fokus auf die Förderung des Gemeinschaftsgefühls durch informelle Treffen legen wollen, wie das folgende Zitat beispielhaft untermauert: «Session 10 Minuten vor und nach dem Termin ohne den Lehrenden/Moderator frei laufen lassen, damit die Teilnehmer sich untereinander unterhalten können.»

\section{Vor welchen Herausforderungen standen Sie mit Ihren Studierenden im SoSe 2020? (n=239)}

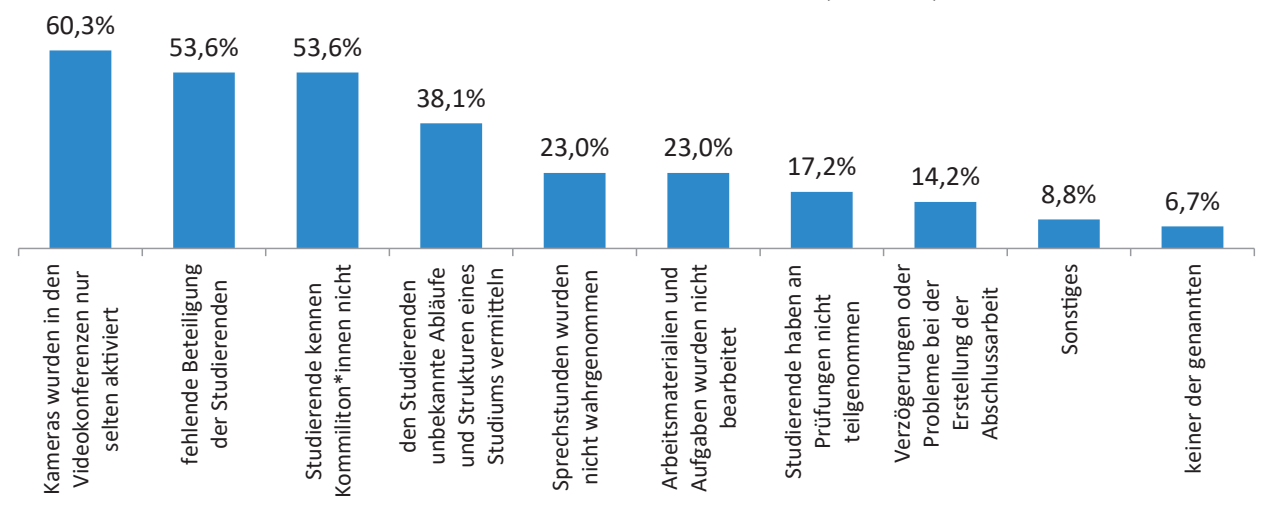

Abb. 3.: Herausforderungen in der Lehre im SoSe 2020.

Die Lehrenden wurden zudem danach befragt, bei welcher Gruppe von Studierenden sie am meisten den Eindruck haben, dass ihr die Teilnahme an Online-Lehrveranstaltungen leichtfalle (FF3). Dabei wurde deutlich, dass es nach Einschätzung der Befragten besonders Studierenden in einem niedrigen Fachsemester schwerfällt, an digitalen Lehrveranstaltungen teilzunehmen (60,1\% fällt es gar nicht oder eher nicht leicht, $n=148$ ). Lehrveranstaltungen für diese Zielgruppe zu konzipieren, ist somit eine weitere Herausforderung für die Lehrenden. Weiterhin gaben die Lehrenden an, inwiefern ihnen die Umsetzung bestimmter Ziele in der Lehre im SoSe 2020 leicht oder schwer fiel (FF3). Zu den Herausforderungen hier gehörte es, die Studierenden persönlich zu betreuen (52\% fiel es gar nicht oder eher nicht leicht, $n=231$ ) beziehungsweise die Bildung studentischer Arbeitsgruppen zu unterstützen (52\% fiel es gar nicht oder eher nicht leicht, $\mathrm{n}=209$ ). 
Abschliessend wurde gefragt, wie sich die Probandinnen und Probanden ihre Lehre in Zukunft vorstellen (FF4). Es lässt sich festhalten, dass sich bei $55,8 \%$ die Bereitschaft erhöht hat, digital gestützte Lehre auch in Zukunft anzubieten ( $n=233)$. Der Grossteil $(44,4 \%)$ möchte eine Kombination aus Präsenz- und Online-Lehre konzipieren ( $n=232$ ). Lediglich $12,1 \%$ der befragten Personen möchten zukünftig reine Online-Lehrveranstaltungen anbieten. $64,0 \%$ planen in Zukunft Screencasts verstärkt einzusetzen ( $n=239$ ). Ebenfalls häufiger zum Einsatz sollen Live-Votings z. B. in Form von Quizzen genauso wie Lernstandskontrollen auf Moodle kommen. Dabei fällt auf, dass deutlich mehr Lehrende angeben, Zwischentests, kollaborative Tools und LiveVotings oder Quizze, in Zukunft einsetzen zu wollen und so mit weiteren digitalen Tools, Lernmaterialien, bzw. Methoden zu arbeiten als sie dies im SoSe 2020 getan haben. Dies ist unter Umständen auf die kurzfristige Umsetzung zurückzuführen und lässt vermuten, dass bei mehr Vorlaufzeit weitere Methoden und Tools eingesetzt würden.

\section{Welche digitalen Tools, Lernmaterialien, bzw. Methoden haben Sie im SoSe 2020 eingesetzt und welche können Sie sich auch in Zukunft im Kontext Ihrer Lehrveranstaltungen vorstellen? $(n=239)$}

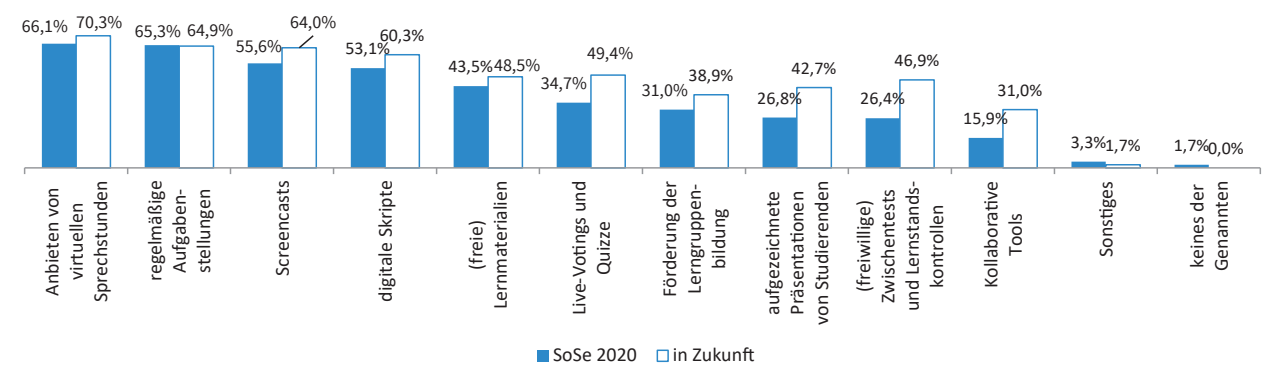

Abb. 4.: Vorstellbare digitale Tools, Lernmaterialien und Methoden.

\section{Limitationen}

Die verschiedenen Methoden haben Einschränkungen, die sich trotz Bemühungen nicht vollständig kompensieren lassen. Die ohnehin begrenzte Anzahl der qualitativen Interviews erschwerte es, alle Statusgruppen zu berücksichtigen. Von den angefragten Lehrbeauftragten kam keine Rückmeldung auf die Interview-Anfrage, so dass diese Statusgruppe bei der qualitativen Teilstudie nicht vertreten ist. Dies ist unter Umständen darauf zurückzuführen, dass Lehrbeauftragte neben ihrer Berufstätigkeit in der Lehre tätig sind und so ein noch geringeres Zeitbudget für Aufgaben rund um die Lehre haben. Da jedoch 36,6\% der standardisierten Fragebögen von Lehrbeauftragten ausgefüllt wurden, hat die Online-Befragung dennoch Einblicke in 
die Perspektive der betroffenen Personen ermöglicht. Auf diese Weise konnten auch über die offenen Fragen die besonderen Herausforderungen dieser Statusgruppe in der Gesamtstudie Berücksichtigung finden.

Eine weitere Limitation kann die soziale Erwünschtheit, insbesondere in den qualitativen Interviews, sein. So ist denkbar, dass einzelne Befragte nicht alle Probleme, die sie mit Online-Lehre haben, benannt haben aus Sorge, dass sie als wenig innovativ oder anpassungsfreudig wahrgenommen werden. In diesem Zusammenhang ist auf die sogenannte «looking-good-Tendenz» (Brosius, Koschel, und Haas 2008, 130) zu verweisen, die davon ausgeht, «dass der Befragte nur Antworten gibt, von denen er denkt, dass sie gut für das Image sind» (Brosius, Koschel, und Haas 2008, 130).

Auch wenn zu erwarten ist, dass bei Online-Befragungen im Response-Verhalten ein hoher Anteil der Lehrenden durch Selbstselektion den Fragebogen nicht ausfüllt, ist die Rücklaufquote mit 21,2\% gerade im Hinblick auf die Belastungen der Lehrenden in der Pandemie-Situation positiv zu deuten. Trotzdem besteht das Problem der willkürlichen Stichprobe, da womöglich gerade diejenigen, die Probleme mit der Online-Lehre haben, nicht geneigt sind, auch noch einen Online-Fragebogen auszufüllen bzw. gerade diejenigen, die ohnehin offen für digital gestützte Lehr- und Lernformate sind, an der Befragung teilnehmen.

In Bezug auf die Auswertung der quantitativen Studie lässt sich kritisieren, dass zunächst nur mit Methoden der deskriptiven Statistik gearbeitet wurden und so keine Aussagen über die Grundgesamtheit getroffen werden können. Für die Zukunft wäre denkbar, vertiefende Analysen mit Mitteln der Inferenzstatistik durchzuführen, um die Befunde zu generalisieren.

Zuletzt ist auf die auseinanderliegenden Befragungszeitpunkte einzugehen. So fand die qualitative Teilstudie noch während des laufenden SoSe 2020 statt, die quantitative Teilstudie jedoch erst nach Ende der Vorlesungszeit. Die Befragten hatten in den qualitativen Interviews daher lediglich erste Erfahrungen im Online-Semester sammeln können und den vollständigen Semesterzyklus noch nicht durchlaufen. D. h. sie kannten die Ergebnisse der Prüfungen noch nicht und konnten so den Lernerfolg der Studierenden in ihren Lehrveranstaltungen nicht endgültig beurteilen. Zum Zeitpunkt der quantitativen Teilstudie bestand diese Limitation nicht mehr, da das Semester bereits abgeschlossen und der Grossteil der Prüfungen absolviert war.

\section{Fazit und Ausblick}

In der Gesamtschau lässt sich sagen, dass die Lehrenden trotz der plötzlich auftretenden Corona-Situation die Lehre im SoSe 2020 insgesamt positiv erlebt haben. So berichten sie von einem grossen Kompetenzzuwachs, was etwa die Interaktion und Kommunikation über Videokonferenzsysteme oder das Erstellen von Medienprodukten angeht. Die Befragten zeigten dabei eine grosse Bereitschaft, sich trotz 
der wenigen Vorbereitungszeit auf reine Online-Lehre einzulassen und setzten dies mit hoher Experimentierfreudigkeit um. Die Experimentierfreudigkeit lässt sich auch daran festmachen, dass der Grossteil der Probandinnen und Probanden vielfältige und aufwändige Lehr- und Lernformen eingesetzt hat (siehe Abschnitt 4). Das besondere Engagement, sich auf die neue Lehrsituation einzustellen und Lehrkonzepte entsprechend anzupassen, führte auch zu einer grösseren Sensibilisierung, was das Thema E-Learning insgesamt angeht. So zeigen sich viele Lehrende interessiert, auch in Zukunft E-Learning-Elemente in ihrer Lehre einzubinden. Wie eingangs von Welzer (1993) beschrieben, können solche Umbruchssituationen folglich auch entwicklungsfördernd und impulsgebend sein.

Die Lehrenden geben zwar an, dass die Konzeption von Online-Lehrveranstaltungen einen erheblichen Mehraufwand mit sich gebracht hat, haben sich jedoch dieser Herausforderung gestellt. Dies gilt sowohl für die hauptamtlich Lehrenden als auch für die Lehrbeauftragten, die insgesamt deutlich weniger Zeit zur Verfügung hatten, da sie häufig nebenberuflich in der Lehre engagiert sind. Dabei wurde klar, dass die Lehrenden in der Tat ihre Lehrkonzepte auf das reine Online-Setting angepasst und umgestaltet haben. Untersuchungen von anderen Hochschulen, wie z. B. die Universität Hamburg (Reinmann et al. 2020), die Ruhr Universität Bochum (RUB 2020) oder die Universität Kiel (Kloschinski et al. 2020) kommen zu einem ähnlichen Ergebnis: Die ad-hoc Umstellung ist für Lehrende mit einem erheblichen Mehraufwand verbunden.

Die zwei grössten Herausforderungen, die Lehrende nennen, sind die Aktivierung und die Unterstützung der Gruppenarbeit von Studierenden. Beiden Herausforderungen haben sie sich mit entsprechenden Tools, Methoden und Lehrszenarien gestellt. Die Befragten geben an, dass sie zukünftig in noch grösserem Masse bereit sind, Live-Votings, Quizze und andere Aktivierungsmethoden einzusetzen als sie es im Rahmen des SoSe 2020 realisieren konnten. Mit etwas mehr Vorbereitungszeit kann also erwartet werden, dass die Vielfalt der eingesetzten Tools, Methoden und Lehrszenarien zur Aktivierung der Studierenden nochmals zunimmt.

Worauf die Lehrenden viel Wert legen, ist synchrone Kommunikation mit den Studierenden. Es hat sich gezeigt, dass rein asynchrone Lehre von den Lehrenden als schwierig betrachtet wird. Dies ist auch darauf zurückzuführen, dass dabei die persönliche Ebene der Kommunikation fehlt. Der so erschwerte Beziehungsaufbau wird dabei von den Befragten sehr bedauert und als grosse Herausforderung gesehen. Dies deckt sich auch mit den aktuellen Befunden der DZHW-Studie zum Corona-Semester, die ebenfalls zu dem Schluss kommt, dass insbesondere der Kontakt zwischen Kommilitoninnen und Kommilitonen und zu Lehrenden als schwieriger wahrgenommen wird (Lörz et al. 2020). Das Fehlen des direkten Austauschs erschwert Lehrenden die Aktivierung der Studierenden. Hier kann die entsprechende Gestaltung der Kennenlernphase und Gruppenarbeiten in Videokonferenzen einen wertvollen Kompensationsbeitrag zum unmittelbaren Austausch leisten. 
Allerdings sind bestimmte Formate nach Ansicht der Befragten gar nicht online umsetzbar. Dazu gehören insbesondere Laborübungen und Exkursionen. Daher überrascht es nicht, dass viele Lehrende betonen, dass sie die Präsenzlehre nicht aufgeben möchten. In den Interviews wurde besonders hervorgehoben, dass es sich bei der Frankfurt University of Applied Sciences um eine Präsenzhochschule handelt. Die grösstenteils positiven Erfahrungen des SoSe 2020 haben die Bereitschaft der Lehrenden vergrössert, in Zukunft eine Kombination aus Präsenz- und Online-Lehre anzubieten bzw. hybride Lehrszenarien zu entwickeln.

Damit sich zum Start des regulären Lehrbetriebs nicht ein abrupter Umstieg zurück auf reine Präsenzlehre vollzieht, könnte es sinnvoll sein, die Lehrenden schon frühzeitig beim Umstieg auf hybride Modelle der Lehre zu unterstützen - etwa durch Workshops, Coachings, Think Tanks oder Peer-Learning-Angebote. Zusätzlich wäre es zielführend, wenn parallel geeignete Rahmenbedingungen wie z. B. die technische Ausstattung in den Räumen an der Hochschule geschaffen werden. Schliesslich setzt hybride Lehre andere technische Bedingungen voraus als reine Online-Lehre.

Die vorliegende Studie hat einen ersten Einblick in die ad-hoc Umstellung auf reine Online-Lehre im SoSe 2020 gewährt. Weitere Untersuchungen in den kommenden Semestern können Aufschluss darüber geben, ob die Absichten und Ziele der Lehrenden realisiert wurden. Zusätzlich könnte ein Vergleich mit der parallel durchgeführten Studierendenbefragungen ein vollständigeres Bild der Online-Lehre zu Beginn der Covid-19-Pandemie geben.

\section{Literatur}

Branch, Robert M., und Tonia A. Dousay. 2015. Survey of Instructional Design Models. k. A.: AECT.

Brosius, Hans-Bernd, Friederike Koschel, und Alexander Haas. 2008. Methoden der empirischen Kommunikationsforschung. Wiesbaden: Springer VS. https://doi.org/10.1007/978-3-53119996-2.

Bryman, Alan. 2008. Social Research Methods. Oxford u. a.: Oxford University Press.

Creswell, John W. 2003. Research design: Qualitative, quantitative and Mixed-Methods approaches. Los Angeles u. a.: Sage.

Dresing, Thorsten, und Thorsten Pehl. 2015. Praxisbuch Interview, Transkription und Analyse. Anleitungen und Regelsysteme für qualitativ Forschende. Marburg: Eigenverlag. https:// www.audiotranskription.de/Praxisbuch-Transkription.pdf.

Friebertshäuser, Barbara. 1997. «Interviewtechniken - ein Überblick». In Handbuch Qualitative Forschungsmethoden in der Erziehungswissenschaft, herausgegeben von Barbara Friebertshäuser, Antje Langer und Annedore Prengel, 371-395. Weinheim: Juventa. 
Friebertshäuser, Barbara, und Antje Langer. 2010. «Interviewformen und Interviewpraxis». In Handbuch Qualitative Forschungsmethoden in der Erziehungswissenschaft, herausgegeben von Barbara Friebertshäuser, Antje Langer und Annedore Prengel, 437-455. Weinheim: Juventa.

Griebel, Wilfried, und Renate Niesel. 2017. Übergänge verstehen und begleiten. Berlin: Cornelsen.

Kelle, Udo. 2014. «Mixed Methods». In Handbuch Methoden der empirischen Sozialforschung, herausgegeben von Nina Baur und Jörg Blasius, 153-166. Wiesbaden: Springer VS. https:// doi.org/10.1007/978-3-531-18939-0.

Kloschinski, Andrea, Daniela Renger, Nora Döring, Anne Döring, Johanna Gerwin, und Ines Weber. 2020. «Forschen und Lehren während der Corona-Pandemie - Auswertung einer Befragung unter Mitarbeiter*innen der CAU Kiel». https://www.phil.uni-kiel.de/de/fakultaet/ beauftragte/GB-PhilFak/aktuelles/auswertung-befragung-corona.

Kuckartz, Udo. 2014. Mixed Methods: Methodologie, Forschungsdesigns und Analyseverfahren. Wiesbaden: Springer VS. https://doi.org/10.1007/978-3-531-93267-5.

Lamnek, Siegfried. 2010. Qualitative Sozialforschung. Lehrbuch. Basel und Weinheim: Beltz.

Lörz, Markus, Anna Marczuk, Lena Zimmer, Frank Multrus, und Sandra Buchholz. 2020. Studieren unter Corona-Bedingungen: Studierende bewerten das erste Digitalsemester. (DZHW Brief 5|2020). Hannover: DZHW. https://doi.org/10.34878/2020.05.dzhw_brief.

Means, Barbara, Marianne Bakia, und Robert Murphy. 2014. Learning Online: What Research Tells Us about Whether, When and How. New York: Routledge.

Meyen, Michael, Maria Löblich, Senta Pfaff-Rüdiger, und Claudia Riesmeyer. 2011. Qualitative Forschung in der Kommunikationswissenschaft. Wiesbaden: Springer VS. https://doi. org/10.1007/978-3-531-92829-6.

Raupach, Tobias, Christian Münscher, Tim Beißbarth, Gerhard Burckhardt, und Tobias Pukrop. 2011. «Towards outcome based programme evaluation: Using student comparative selfassessments to determine teaching effectiveness» Med Teach 33, Nr. 8: S. 446-453. https:// doi.org/10.3109/0142159x.2011.586751.

Raupach, Tobias und Sarah Schiekirka. O. J.: Handreichung zur Lernerfolgsevaluation. Electric Paper Evaluationssysteme GmbH.

Reinmann, Gabi. 2013. Didaktisches Handeln. Die Beziehung zwischen Lerntheorien und Didaktischem Design. https://nbn-resolving.org/urn:nbn:de:0111-opus-83381.

Reinmann, Gabi, Carla Bohndick, Eileen Lübcke, Alexa Brase, Marikje Kaufmann, und Nele Groß. 2020. «Emergency Remote Teaching im Sommersemester 2020. Bericht zur Begleitforschung - Lehrendenbefragung». https://www.hul.uni-hamburg.de/forschung/projektarchiv/ert/begleitforschung-bericht-2020-2.pdf.

Ruhr-Universität Bochum. 2020. «Erste Ergebnisse der Lehrendenbefragung zur online-basierten Lehre im Sommersemester 2020». https://ruhr-uni-bochum.sciebo.de/s/PLqkMqiID8Y8QjA.

Welzer, Harald. 1993. Transitionen. Zur Sozialpsychologie biographischer Wandlungsprozesse. Tübingen: Edition diskord. 